Int. J. Morphol.,

31(1):128-130, 2013.

\title{
Digestive Peritoneum in Wistar Rat (Rattus norvegicus)
}

\author{
Peritoneo del Aparato Digestivo de la Rata Wistar (Rattus norvegicus)
}

\author{
Richard Möller; Noelia Vazquez; Diana Teliz \& Virginia Méndez
}

MÖLLER, R.; VAZQUEZ, N.; TELIZ, D. \& MÉNDEZ, V. Peritoneo del aparato digestivo de la rata wistar (Rattus norvegicus). Int. J. Morphol., 31(1):128-130, 2013.

RESUMEN: La rata de laboratorio (Rattus norvegicus albinus) ha sido usada como modelo para investigaciones médicas, biológicas y moleculares, desde hace mucho tiempo. Sin embargo, no existen descripciones detalladas del peritoneo digestivo de la rata wistar. El objetivo de este trabajo es definir en forma clara y acorde a los principios de la Nómina Anatómica Veterinaria el peritoneo en esta especie de mamífero de laboratorio. Se utilizaron ochenta y ocho ratas (Rattus norvegicus albinus) de entre 250 y 450 g. Fueron disecadas en fresco luego de ser eutanasiadas por sobredosis de tiopental sódico intraperitoneal. Se realizó una incisión paramediana que se extendía desde el cartílago xifoides del esternón hasta el pubis y otra sobre cada arco costal hacia dorsal.

PALABRAS CLAVE: Anatomía; Morfología; Mamíferos; Rattus norvegicus.

\section{INTRODUCCIÓN}

La rata de laboratorio (Rattus norvegicus albinus) ha sido usada como modelo para investigaciones médicas, biológicas y de diversa índole desde hace mucho tiempo. Es la especie más empleada en investigación biomédica teniendo en cuenta el volumen de información existente acerca de ella. Es interesante el hecho de que no existen descripciones detalladas del peritoneo digestivo de esta especie pese a que existen varios artículos y libros sobre su morfología (Chiasson, 1969; Greene, 1963, Langer, 2002). Lo mismo sucede para la descripción de las demás partes del aparato digestivo (Möller \& Vázquez, 2010, 2011; Ofusori \& Caxton-Martins, 2008; Ofusori et al., 2008; Robert, 1971; Sharp \& La Regina, 1998). El objetivo de nuestro trabajo es describir el peritoneo digestivo en la rata de laboratorio, acorde a los principios de la Nómina Anatómica Veterinaria (International Committee on Veterinary Gross Anatomical Nomenclature, 2005).

\section{MATERIAL Y MÉTODO}

Se utilizaron 88 ratas (Rattus norvegicus albinus) de entre 250 y $450 \mathrm{~g}$ criadas en condiciones acordes con el Manual sobre el cuidado y uso de los animales de experimentación (Consejo Canadiense de Protección de los Animales, 1998). Fueron disecadas en fresco luego de ser eutanasiadas por sobredosis de tiopental sódico intraperitoneal. Se abordó el abdomen realizando por una incisión con bisturí en una línea paramediana y luego se practicaron dos incisiones sobre el arco costal hacia dorsal para tener una amplia visión de la cavidad abdominal. Los distintos tramos del aparato digestivo fueron identificados in situ para permitir el reconocimiento de las partes del peritoneo que están relacionadas a ellos. Posteriormente las vísceras abdominales fueron extraídas para completar el estudio.

\section{RESULTADOS}

El estómago estaba fijado cranealmente por el omento menor que se extendía desde la curvatura menor del estómago hasta el hilio del hígado. El omento menor presentaba poca grasa y se observaban bien definidos el ligamento hepato-gástrico y el ligamento hepato-duodenal. Asimismo el ligamento hepato-gástrico se observó dividido en dos partes: una fijada sobre el hilio del hígado (15) y la otra sobre la cara visceral del lóbulo lateral izquierdo $\left(15^{*}\right)$. El omento mayor seoriginaba dorsalmente sobre la línea media, se observaba con grasa, replegado sobre la curvatura mayor del estómago y presentaba un foramen omental bien marcado. El duodeno estaba sujeto por el mesoduodeno que tenía su origen hacia la derecha de la raíz del mesenterio. El pliegue duodenocólico se extendía entre la parte ascendente del 


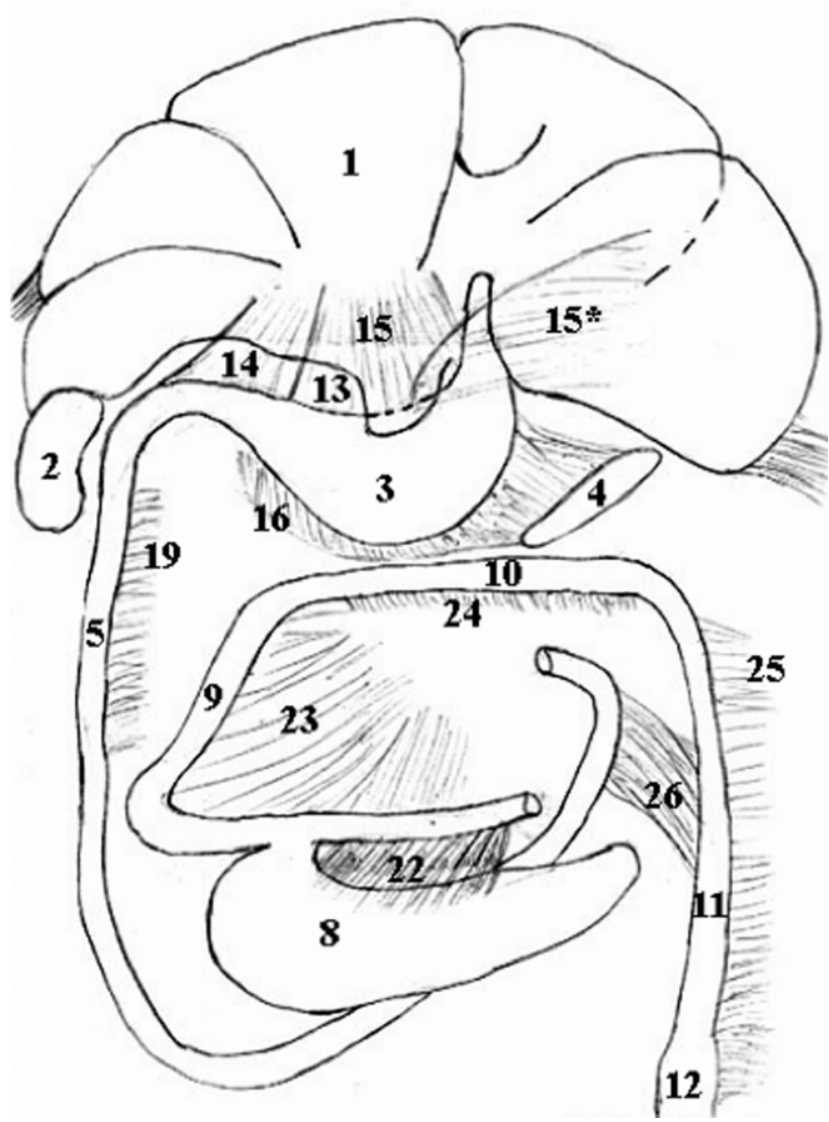

Fig. 1. Esquema donde se observan: 1 hígado, 2 riñón derecho, 3 estómago, 4 bazo, 5 duodeno, 8 ciego, 9 colon ascendente, 10 colon transverso, 11 colon descendente, 12 recto, 13 omento menor, 14 ligamento hepatoduodenal, 15 y $15 *$ ligamento hepatogástrico, 16 omento mayor, 19 mesoduodeno, 22 pliegue ileocecal, 23 mesocolon ascendente, 24 mesocolon transverso, 25 mesocolon descendente, 26 pliegue duodenocólico.

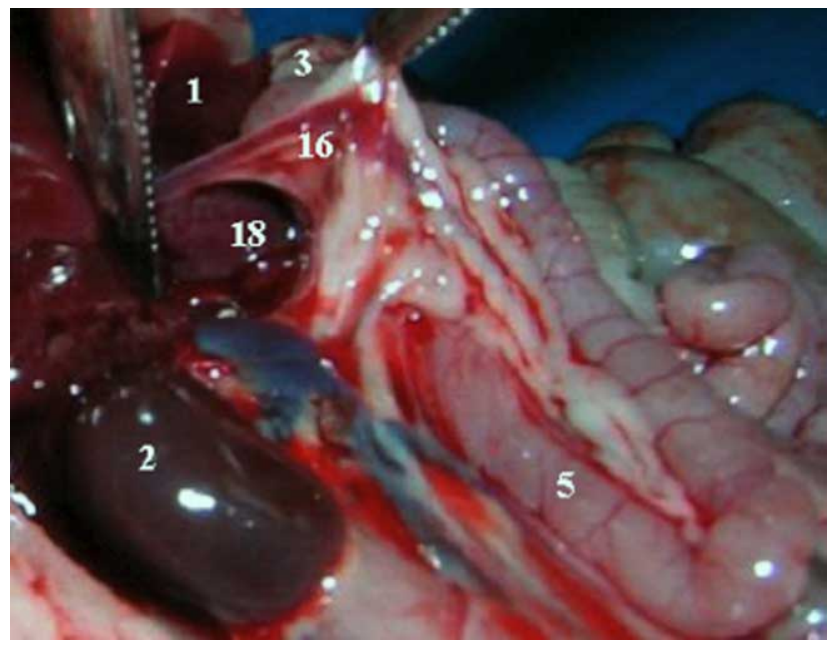

Fig. 2. En la imagen se observan: 1 hígado, 2 riñón derecho, 3 estómago, 5 duodeno, 16 omento mayor, 18 foramen omental. duodeno y la hoja derecha del mesocolon descendente. La raíz del mesenterio daba origen al mesoyeyuno y al mesoíleon desde donde derivaba la sujeción para el ciego, continuándose como pliegue ileocecal. El mesocolon ascendente se originaba hacia la derecha del mesenterio y el colon transverso estaba fijado por un corto mesocolon cranealmente a la raíz del mesenterio. El colon descendente se encontraba sujeto por el mesocolon descendente y la parte craneal del recto por el mesorrecto.

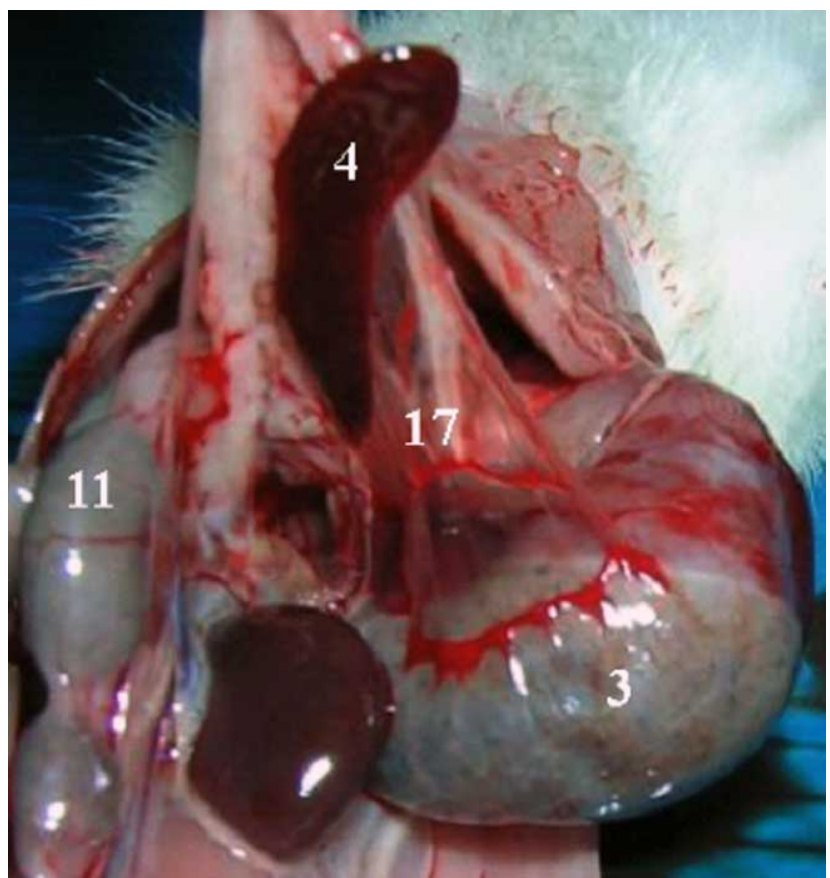

Fig. 3. En la imagen se observan: 4 bazo, 11 colon descendente, 17 ligamento gastroesplénico.

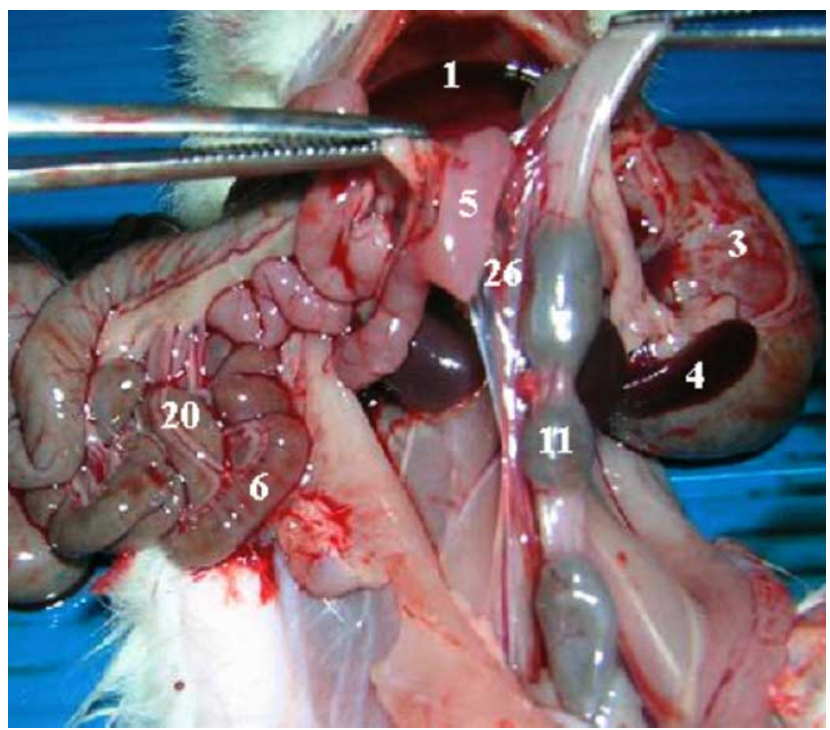

Fig. 4. En la imagen se observan: 1 hígado, 3 estómago, 4 bazo, 5 duodeno, 6 yeyuno, 11 colon descendente, 20 mesoyeyuno, 26 pliegue duodenocólico. 


\section{DISCUSIÓN}

Si bien la rata es uno de los animales más usados como reactivo biológico o como animal de experimentación, los detalles del peritoneo no han sido descritos en la bibliografía disponible (Chiasson; Greene; Langer). Sin embargo existen diferencias en su disposición derivadas de las variaciones en las partes del aparato digestivo descritas por Möller et al. $(2010,2011)$. No obstante, cabe destacar que el ligamento hepato-gástrico se divide en dos partes lo cual es una diferencia interesante respecto de los mamíferos domésticos. Se concluye que el peritoneo digestivo de la rata es similar al de los mamíferos domésticos con variaciones y adaptaciones a la disposición del aparato digestivo.

\section{AGRADECIMIENTOS}

Expresamos nuestro más profundo agradecimiento al Prof. Dr. Álvaro Freire por proveernos de los animales.

MÖLLER, R.; VAZQUEZ, N.; TELIZ, D. \& MÉNDEZ, V. Digestive peritoneum in wistar rat (Rattus norvegicus). Int. J. Morphol., 31(1):128-130, 2013.

SUMMARY: The laboratory rat (Rattus norvegicus albinus) has been used as a model for medical, biological and molecular research, for a long time. It is an interesting fact that there are no detailed descriptions of the gross anatomy of the liver and its ways of fixation. The aim of this study is to define clearly and according to the Nomina Anatomica Veterinaria principles, the liver and its way of fixation in this species of laboratory mammal. Eighty-eight rats (Rattus norvegicus albinus) were used, with a weight between 250 and $450 \mathrm{~g}$. They were dissected in fresh, after been euthanized by an overdose of thiopental sodium intraperitoneal. An incision from the xiphoid cartilage up to the pubic region was made and another on each costal arch toward dorsal.

KEYWORDS: Anatomy; Morphology; Mammals; Rattus norvegicus.

\section{REFERENCIAS BIBLIOGRÁFICAS}

Chiasson, R. Laboratory anatomy of the white rat. 2. ed. Arizona, Wm. C. Brown, 1969. p.81.

Consejo Canadiense de Protección de los Animales. Manual sobre el cuidado y uso de los animales de experimentación. Ottawa, Bradda Printing Services, 1998. pp.2, 323.

Greene, E. Anatomy of the rat. New York, Hafner, 1963.

Langer, P. The digestive tract and life history of small mammals. Mammal. Rev., 32(2):107-31, 2002.

International Committee on Veterinary Gross Anatomical Nomenclature. Nómina Anatómica Veterinaria. $5^{\text {th }}$ ed. Editorial Committee Hannover, Columbia, Gent, Sapporo, 2005.

Möller, R. \& Vázquez, N. Divisions of the digestive tract in wistar rat (Rattus norvegicus). Vet. Zootec., 17(3):415-20, 2010 .

Möller, R. \& Vázquez, N. Anatomy of the Liver in Wistar Rat (Rattus norvegicus). Int. J. Morphol., 29(1):76-9, 2011.

Ofusori, D. \& Caxton-Martins, E. A Comparative Histomorphometric Study of the Stomach of Rat (Rattus norvegicus), Bat (Eidolon helvum) and Pangolin (Manis tricuspis) in Relation to Diet. Int. J. Morphol., 26(3):66974, 2008.
Ofusori, D.; Caxton-Martins, E.; Komolafe, O.; Oluyemi, K.; Adeeyo, O.; Ajayi, S.; et al. Comparative Study of the Ileum in Rat (Rattus norvegicus), Bat (Eidolon helvum) and Pangolin (Manis tricuspis) as Investigated Using Histological Method. Int. J. Morphol., 26(1):137-41, 2008.

Robert, A. Proposed terminology for the anatomy of the rat stomach. Gastroenterology, 60(2):344-5, 1971.

Sharp, P. \& La Regina, M. The Laboratory rat. Boca Raton, Ed. CRC Press, 1998.

Dirección para correspondencia:

Dr. Richard Möller

Área de Anatomía

Departamento de Morfología y Desarrollo

Facultad de Veterinaria

Universidad de la República

Lasplaces 1550, Montevideo. C.P. 11600

URUGUAY

Email: anatomia.uy@gmail.com

Received: 29-05-2012

Accepted: 10-10-2012 